\title{
ACCRETION DISK INSTABILITIES
}

\author{
S. MINESHIGE \\ Department of Physics, Ibaraki University, Mito 310, Japan
}

\begin{abstract}
Basic properties of accretion disk instabilities are summarized. We first explain the standard disk model by Shakura and Sunyaev. In this model, the dominant sources of viscosity are assumed to be chaotic magnetic fields and turbulence in gas flow, and the magnitude of viscosity is prescribed by so-called $\alpha$ model. It is then possible to build a particular disk model. In the framework of the standard model, accretion disks are stationary, but when some of the basic assumptions are relaxed, various kinds of instabilities appear. In particular, we focus on the thermal limit-cycle instability caused by partial ionization of hydrogen (and helium). We demonstrate that the disk instability model well accounts for the basic observed features of outbursts of dwarf novae and X-ray nova. We then introduce other kinds of instabilities based on the $\alpha$ viscosity model. They are suspected to produce time variabilities observed on a wide range of timescales in close binaries and active galactic nuclei.
\end{abstract}

\section{Introduction}

It is widely believed that accretion disks play important roles in a variety of astrophysical objects, including active galactic nuclei (AGN), close binary systems, such as cataclysmic variables (CVs) and X-ray binaries (XBs), and protoplanetary systems. Disk accretion is indeed a very efficient mean to convert rest-mass energy of infalling material into radiation energy; huge energy output is thus possible in AGN disks. Observed high-energy phenomena in XBs and AGN are often interpreted as the activities of disks or disk coronae. Protoplanetary disks provide places for planet formation. Moreover, jets or winds in AGN and protostars seem to be generated in the inner portions of the accretion disks.

Suppose a point mass star with gravitational potential, $\Phi=G M / r$ (where $M$ is the mass of the central star and $r$ is the distance from the star), embedded in gas clouds. If gas particles in ambient clouds are at rest with respect to the central star; namely, when clouds have no systematic angular momentum, gas particles will directly fall onto the central star due to the gravitational force. This is a case of spherical accretion.

If a gas particles in clouds are rotating around the star in the same direction, i.e., if a gas cloud has an angular momentum, on the other hand, gas cannot directly accrete onto the star, but it will rotate around the star on an eccentric orbit. The orbit of each gas particle is characterized by its semi-major axis, $a$, and its specific angular momentum, $l$. After a few rotation, each gas orbit is expected to be circularized by losing eccentricity through collision between particles. The formation of a circular ring or disk is therefore highly plausible. The radius of the circular ring, the circularization radius, is related to its specific angular momentum by, 
$r_{\text {circ }}=\frac{l^{2}}{G M}$.

To sum up, the coexistence of a deep potential well and ambient gas clouds with an angular momentum with respect to the central potential well leads to the formation of a rotating ring or disk.

This is, however, a rotating disk and not an "accretion disk." For rotating gas to accrete onto the central star, each gas particle should somehow lose its angular momentum. We are now encountered with the fundamental issue in the theory of viscous accretion disks; viscosity provides a mechanism for angular momentum transport and heating of plasmas, but the ordinary molecular viscosity is too small to cause mass accretion in reasonable timescales (less than the age of the universe). We therefore require that disk plasmas be turbulent and/or full of chaotic magnetic fields. Turbulence and magnetic fields are dominant sources of viscosity. This is the basic assumption adopted in the theory of accretion disks (Pringle and Rees 1972; Shakura and Sunyaev 1973; Novikov and Thorne 1973; for a review, see Pringle 1981).

The plan of this review paper is as follows: $\S 2$, we explain the standard model (hereafter referred to as SS model). In this model, disks are assumed to be quasi-steady. However, by relaxing some of basic assumptions of SS model, we can derive various kinds of disk instabilities, which will manifest themselves as some observable effects. The most successful example as such is the thermal limit-cycle instability discussed in $\S 3$. This instability is caused by partial ionization of hydrogen and helium and is thought to trigger outbursts of CVs and LMXBs. Other kinds of instabilities are briefly reviewed in $\S 4$. Final section is devoted to summary.

\section{Standard Disk Model}

\subsection{Basic Assumptions}

The standard accretion disk model by Shakura and Sunyaev (1973) is constructed on the following assumptions:

(a) Gas in the disk is rotating around a central star on a circular orbit with the local Keplerian velocity given by,

$$
v_{\varphi}=\sqrt{\frac{G M}{r}},
$$

and gradually moves inward with radial velocity, $v_{\mathbf{r}}$,

$$
v_{r} \ll v_{\varphi},
$$

by losing angular momentum. Here we use the cylindrical coordinate, $(r, \varphi, z)$ with the $z$ axis perpendicular to the disk plane and the $r$ axis in the central 


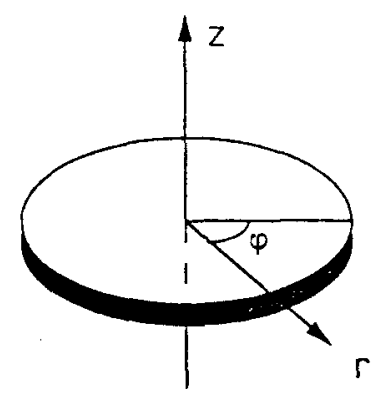

Fig. 1. Cylindrical coordinate, $(r, \varphi, z)$, used in this review.

plane; $z=0$ (see Fig. 1). If we define the viscous timescale and the dynamical timescale as

$$
t_{\mathrm{vis}} \equiv \frac{r}{v_{\mathrm{r}}}, \quad t_{\mathrm{dyn}} \equiv \frac{r}{v_{\varphi}}
$$

we find from equation (3)

$$
t_{\text {vis }} \gg t_{\text {dyn }} \text {. }
$$

(b) The disk is in stationary state in the sense that the mass inflow rate,

$$
\dot{M}=-2 \pi r v_{\mathrm{r}} \Sigma,
$$

(which is positive for inflow) is constant in space and time, where

$$
\Sigma=\int_{-\infty}^{\infty} \rho d z
$$

is surface density.

(c) Disks are geometrically thin,

$$
H \ll r \text {, }
$$

where $H$ is the half thickness of the disk. Typically we find $H / r \sim 1 / 30$. It is then reasonable to use one-zone approximation to describe the vertical structure of the disk, in which all the derivatives with respect to $z$ are replaced by $1 / H$. Furthermore, we can safely decouple two-dimensional equations (in $r$ and $z$ directions) into a set of two, separate, one-dimensional equations: those describing the radial $(r)$ structure and those for the vertical (z) structure.

(d) Hydrostatic balance holds in the vertical ( $z$ ) direction. This condition is under one-zone approximation written as,

$$
\frac{P}{H}=\rho g_{z},
$$


where $g_{z}$ is the vertical component of the gravity at the surface of disk $(z=H)$ due to the central star, and is

$$
g_{z} \simeq \frac{G M}{r^{2}} \frac{H}{r}=\Omega^{2} H,
$$

$\rho$ is the mean density of the disk, and $\Omega$ is the Keplerian angular frequency,

$$
\Omega=\frac{v_{\varphi}}{r}=\sqrt{\frac{G M}{r^{3}}} .
$$

(e) The disk is optically thick;

$$
\tau \gg 1
$$

where

$$
\tau=\left(\kappa_{\text {es }}+\kappa_{\text {abs }}\right) \rho H,
$$

and $\kappa_{\text {es }}$ and $\kappa_{\text {abs }}$ are opacities due to electron scattering and absorption, respectively. The emergent energy flux from a unit area of the surface, which determines the cooling rate of the disk, is then

$$
F=Q^{-}=\frac{4 \sigma T^{4}}{3 \tau},
$$

where $\sigma$ is the Stefan-Boltzmann constant and $T$ is the mean disk temperature (usually represented by a value at $z=0$ ).

(f) The $r-\varphi$ component of the shear stress tensor is prescribed as

$$
\sigma_{r \varphi}=-\alpha P \text {, }
$$

where local pressure $P$ is given by the sum of gas pressure and radiation pressure;

$$
P=P_{\text {gas }}+P_{\text {rad }}
$$

and $\alpha$ is the viscosity parameter $(\alpha<1)$. Viscosity in the accretion disk plays two important roles: angular momentum transport and heating of disk plasmas. The heating rate of the disk is then

$$
Q^{+}=\frac{3}{4} W \Omega,
$$

where $W$ is vertically integrated viscous stress,

$$
W \equiv \int_{-\infty}^{\infty} \alpha P d z
$$

(g) Finally we assume energy balance. Potential energy of accreting gas is first converted to thermal energy via viscous process, and then is released as radiation. Therefore, local energy balance at each radius is given by

$$
Q^{+}=Q^{-}
$$

where heating is due to viscous heating [eq. (17)] and cooling is via blackbody radiation [eq. (14)]. 


\subsection{Stationary Relations}

Stationary conditions for radial disk structure are derived from the following basic equations;

$$
\begin{aligned}
& \frac{\partial \Sigma}{\partial t}=\frac{1}{2 \pi r} \frac{\partial \dot{M}}{\partial r}, \\
& \dot{M} \sqrt{\frac{G M}{r}}=4 \pi \frac{\partial}{\partial r}\left(r^{2} W\right),
\end{aligned}
$$

where the former equation represents mass conservation and the latter describes angular momentum transport.

By setting $\partial / \partial t=0$ in equation (20), we have constant $\dot{M}$ and

$$
F=\frac{3}{8 \pi} \frac{G M \dot{M}}{r^{3}}\left[1-\sqrt{\frac{r_{*}}{r}}\right]
$$

where $r_{*}$ is the radius of the inner edge of the disk and we used relations (19) and (21). The emergent local flux is hence independent of the magnitude of the viscosity, and is proportional to $r^{-3}$ at $r \gg r_{\star}$. The larger $\alpha$ is, the smaller becomes the disk mass, $M_{\mathrm{d}}$, and so does surface density, $\Sigma$. For a given mass-input rate, however, the total energy emitted locally, $F$, and the total disk luminosity, $L_{\mathrm{d}}$, are independent of $\alpha$, because increase (decrease) in radial flow velocity caused by increase (decrease) in the $\alpha$ value totally compensates with the decrease (increase) in the disk mass.

Now we obtain expressions for physical quantities, such as $T, H, \Sigma, W$, $\tau$, as functions of radius $r$ for given parameters $M, \dot{M}$, and $\alpha$.

Effective temperature of the disk, defined as

$$
T_{\text {eff }} \equiv(F / \sigma)^{1 / 4} \propto r^{-3 / 4} \text { at } r \gg r_{\star},
$$

has radial dependence in accretion disks (see Fig. 2). For fixed $M$ and $r, T_{\text {eff }}$ increase as $\dot{M}$ increases.

\subsection{Comparison Between Disk Models and Stellar Atmosphere MODELS}

As we have seen previously, vertical disk structure is in many respects analogous to stellar atmosphere structure; e.g., gas layer is in hydrostatic balance, excess energy is carried towards the surface by radiation, and local energy balance holds. There are, however, some discrepancies between them:

(a) One single star has only one value of $T_{\text {eff }}$ under spherically symmetric assumption, whereas $T_{\text {eff }}$ in a stationary disk is radially dependent. Roughly speaking, an accretion disk is composed of ensemble of different types of stellar atmospheres with radially different $T_{\text {eff }}$ and surface gravity $g$. (Note, 


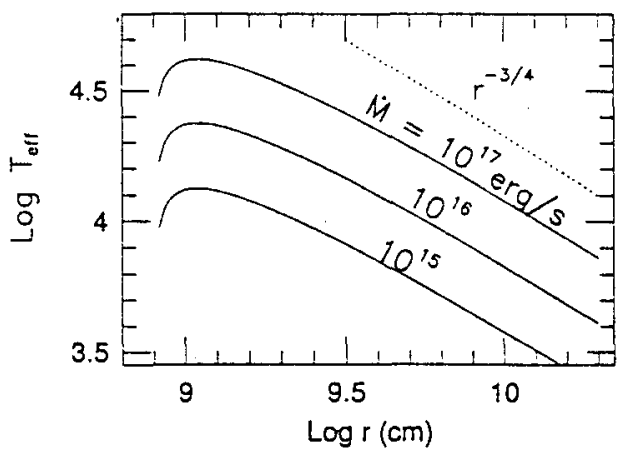

Fig. 2. Effective temperature distribution of the stationary disks for given values of $\dot{M}=10^{15}, 10^{16}$ and $10^{17} \mathrm{erg} \mathrm{s}^{-1}$. Here the mass of a central star is $M=1 M_{\odot}$.

however, disk structure is not exactly equal to that of stellar atmospheres; see below.) Disk spectra are thus composed of multi-color spectra.

(b) Energy generation in a star takes place only at a core. Hence energy generation rate is practically zero, $\epsilon=0$, in stellar atmospheres. In contrast, viscous heating is everywhere available in a disk, so $\epsilon \neq 0$ in the disk structure (see eq. 17 and 18 ).

(c) Gravity is nearly constant in stellar atmospheres, whereas $g_{z}(z) \propto z$ in a disk.

\section{Thermal Limit-Cycle Instability}

Now we are at the position to discuss the stability of accretion disks based on the $\alpha$ model. We first describe the thermal limit-cycle instability, which has most successively linked the theory of time-dependent accretion disk models and the observed time variabilities in close binary systems.

\subsection{Partial Ionization of Hydrogen in The Disk}

It is well known that the partial ionization process of hydrogen in stars provides so-called $\kappa$-mechanism for stellar pulsation (see, e.g., a review by Saio 1992 in this volume). When plasma temperature is low enough for hydrogen to recombine, the opacity $\kappa$ changes quite rapidly with change in temperature; $\kappa \propto T^{a}$ with $a=5-10$. The outgoing radiation is thus efficiently blocked in a compressed phase of stellar pulsation because of enormous increase in $\kappa$ in higher temperature regime compared with the value in an expanded phase, leading to overstable oscillation. Partial ionization process of hydrogen thus manifests itself as visible effects in stars.

The question then arises as to what visible phenomenon is expected in accretion disk when partial ionization process of hydrogen sets out. Rapid 
ulanges in opacity due to ionization and recombination of hydrogen and helium are very likely to produce some visible effects in disks as well, because hydrogen is most abundant in disks and the partial ionization process is a basic atomic process.

The answer to this question is the thermal limit-cycle instability, which yields sporadic light variation in disk luminosity. It is now widely believed that this mechanism causes outbursts of dwarf novae (DN), a subgroup of CVs, (Hoshi 1979; Meyer and Meyer-Hofmeister 1981; Cannizzo et at 1982; see also Smak 1984a for a review), and those of soft X-ray transients (or $\mathrm{X}$-ray novae, XN), a subgroup of low mass X-ray binaries (e.g., Mineshige and Wheeler 1989; Mineshige et al. 1990).

\subsection{S-Shaped Thermal Equilibrium Curves}

To study time-dependent properties of unstable accretion disks, we need to vertically integrate the disk structure, because the presence of vertical convection is a key to understand the instability (Meyer and Meyer-Hofmeister 1981, 1982; Faulkner et at 1983; Smak 1982a; Mineshige and Osaki 1983). Note that one-zone approximation, introduced in $\S 2$, is valid only for radiative disks, where convective energy transport is negligible.

Basic differential equations for vertical structure are equations of continuity,

$$
\frac{d \Sigma_{z}}{d z}=-2 \rho
$$

hydrostatic balance,

$$
\frac{d P}{d z}=-\rho g_{z}
$$

energy balance,

$$
\frac{d F}{d z}=\frac{3}{2} \alpha P \Omega
$$

and energy transport,

$$
\frac{d \ln P}{d \ln T}=\nabla_{\mathrm{rad}} \text { or } \nabla_{\mathrm{conv}}
$$

with supplementary equations

$$
\begin{aligned}
& \frac{d \tau}{d z}=-\kappa \rho, \\
& \frac{d W}{d z}=-\alpha P,
\end{aligned}
$$


where $\Sigma_{z}(z)$ is surface density integrated from the surface $(z=H)$ to $z, F_{z}$ is energy flux at $z$, and $\nabla_{\text {rad }}$ and $\nabla_{\text {conv }}$ are pressure gradient for radiative energy transport and convective energy transport, respectively. Convective gradient is calculated by the mixing-length theory (Cox and Giuli 1968; Paczyński 1969) and Rosseland-mean opacity, $\kappa$, is taken from the tables by Cox and Stewart (1970) and Alexander et al. (1983).

We integrate these equations from the surface at $z=H$ (where $H$ is a parameter) with the surface boundary conditions,

$$
\begin{aligned}
& \Sigma_{z}=0, T=T_{\text {eff }}, \quad F_{z}=\sigma T_{\text {eff }}^{4}, \\
& \tau_{1}=2 / 3, \text { and } \kappa\left(\rho_{1}, T_{\text {eff }}\right) P\left(\rho_{1}, T_{\text {eff }}\right)=\tau_{1} g_{z}(H),
\end{aligned}
$$

towards the center $(z=0)$. Here the last relation in eq.(30), derived from the combination of equations (25) and (28), gives a condition for density at the surface, $\rho_{1}$. The value of the scale height $H$ is chosen so that the following central boundary conditions are to be met,

$$
F_{z}=0, \text { at } z=0 \text {. }
$$

For fixed values for $r, \alpha$, and $M$, we get a family of solutions as functions of $\dot{M}$. Figure 3 schematically displays equilibrium relation between the mass flow rate out of a ring at $r, \dot{M}_{\text {out }}$ (see Fig. 4 ) and $\Sigma$. The essence of the thermal instability is summarized in this S-shaped equilibrium curve. There exist three solutions for a certain range of $\Sigma ; \Sigma_{B}<\Sigma<\Sigma_{A}$. Hydrogen is fully ionized in the high state (HII state), and it is neutral in the low state (HI state). These two branches are thermally and viscously stable (see, e.g., Mineshige and Osaki 1983), whereas the middle state, in which hydrogen is partially ionized, turns out to be unstable against thermal and viscous instabilities. When the mass input rate into a ring, $\dot{M}_{\text {in }}$, falls onto a range, $\dot{M}_{A}<\dot{M}<\dot{M}_{B}$ (see Fig. 3), limit-cycle behavior of the disk unavoidably appears. The presence of such limit cycles was intuitively predicted by Osaki (1974).

Suppose the disk lying in the low state. Since $\dot{M}_{\text {in }}>\dot{M}_{\text {out }}$ in this state, $\Sigma$ (and disk mass) should increase. The evolutionary track of such an unstable disk follows the low equilibrium line up to point $B$ as indicated by an arrow in Fig. 3. Since there is no low state at $\Sigma>\Sigma_{B}$ and $Q^{+}>Q^{-}$on the right side of the $S$ shape, disk temperature will increase. Hydrogen in the disk starts to get ionized and eventually the disk jumps to the HII hot state, where $\dot{M}_{\text {out }}>\dot{M}_{\text {in }}$ holds. The disk mass (or $\Sigma$ ) should decrease there, as the disk gas falls onto the central star. At point A, the thermal instability again takes place and the disk falls into the HI cool state. All the hydrogen in the disk is neutral there. Now one limit cycle is completed.

In summary, any accretion disks with mass-input rate in a range, 


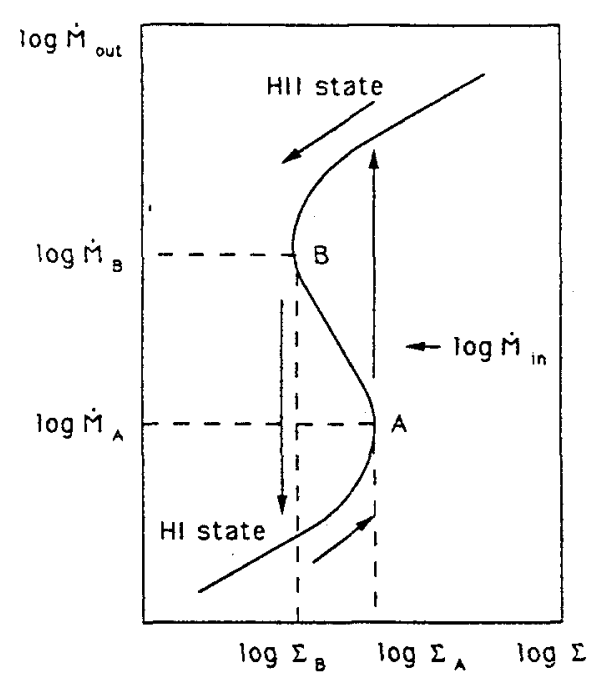

Fig. 3. Schematic thermal equilibrium curve in dwarf-nova disks. Hydrogen is fully ionized (HII) on the upper branch, partially ionized on the middle branch, and are neutral (HI) on the lower branch. The middle solution between points $A$ and $B$ is thermally unstable. When the mass input rate falls onto a range between $\dot{M}_{A}$ and $\dot{M}_{B}$, the disk alternates between two stable states, as indicated by arrows.

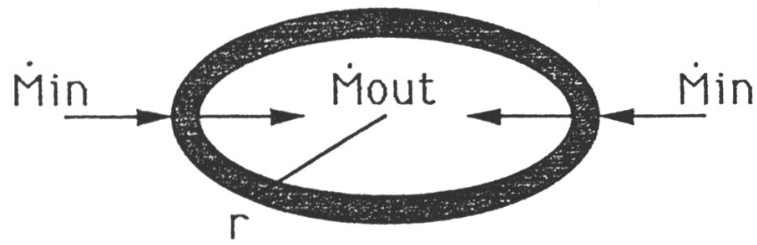

Fig. 4. Mass outflow, $\dot{M}_{\text {out }}$, from a ring at radius $\tau$ and mass inflow, $\dot{M}_{\text {in }}$, into a ring.

$$
\dot{M}_{A}<\dot{M}_{\text {in }}<\dot{M}_{B}
$$

inevitably produces $L_{\mathrm{d}}$ variation due to the relaxation oscillations of the disk between HI and HII states.

Smak (1982b) plotted $\dot{M}$ and $r_{\mathrm{d}}$ for some individual CVs, and found that those CVs with $M$ satisfying the criterion (30) at $r=r_{\mathrm{d}}$ exhibit DN outbursts, while those with $\dot{M}>\dot{M}_{B}$ have no outbursts and are thus classified as nova-like objects. This is a direct evidence for the disk-instability model for outbursts of DN. A similar plot was attempted for XN by Cannizzo et al. (1985), who confirmed the same result for X-ray binaries.

\subsection{Time Evolution of Unstable Disks}

Local behavior of the unstable disk is characterized by the thermal limit cycles. Next, we need to simulate the global response of the local instability. The time-dependent basic equations for radial disk structure are equations 
(20), (21), and vertically averaged energy equation;

$$
\frac{C_{p} \Sigma}{2}\left(\frac{\partial T}{\partial t}+v_{\mathrm{r}} \frac{\partial T}{\partial r}\right)=Q^{+}-Q^{-}+\frac{H}{r} \frac{\partial\left(r F_{\mathrm{r}}\right)}{\partial r},
$$

where $F_{\mathrm{r}}$ is the radial heat flux;

$$
F_{\mathrm{r}}=\frac{4 a c T^{3}}{3 \kappa \rho} \frac{\partial T}{\partial r} \text {. }
$$

They are diffusion type equations for gas (eqs. 20 and 21) and for heat (eqs. 33 and 34). By means of time dependent simulations, we can see how local instability is spatially propagated (Papaloizou et al. 1983; Smak 1984b; Mineshige and Osaki 1985; Cannizzo et al. 1986).

Note that this is a typical example of the dissipative structure (Nicolis and Prigogine 1977). We may find good examples of the dissipative structure in various contexts of astrophysical plasmas. Spatial pattern formation of three-phased interstellar medium is discussed by Tainaka et al. (1992) in this volume.

The results of transition wave propagation are displayed in Figure 5, which is taken from Mineshige (1987). Note that sharp peaks in the heating front in $\Sigma$ diagram look like shock feature, but front speed is $v_{F} \sim \alpha C_{\mathbf{s}}$ (where $C_{\mathrm{s}}$ is the sound velocity), and is subsonic (recall $\alpha<1$; Meyer 1984).

The mass accretion rate at $r=r_{*}$, the $\mathrm{V}$-magnitude, and the total disk luminosity (Mineshige and Wheeler 1989).

$$
L_{\mathrm{d}}=\int_{r_{*}}^{r_{\mathrm{d}}} 2 \cdot F(r) 2 \pi r d r
$$

is depicted in Fig. 6 for the case of X-ray nova (Mineshige and Wheeler 1989). The basic features of the theoretical light curves calculated based on the disk instability model are in good agreement with those of the observed $\mathrm{X}$-ray and optical light curves of DN and XN.

Finally the same mechanism could operate in the disks in galactic nuclei and may be responsible for AGN phenomena (Mineshige and Shields 1990). It is also suggested that the thermal limit cycles cause FU Ori eruptions in protostar systems (Lin and Papaloizou 1985).

\section{Other Disk Instabilities}

In this section, we briefly introduce the fundamentals of other disk instabilities together with some observational implications.

\subsection{Instabilities in Radiation-Pressure Dominated Disks}

As long as the $\alpha$ prescription (15) is adopted, accretion dishs are thermally and viscously unstable when radiation pressure, $P_{\text {rad }}$, dominates over gas 

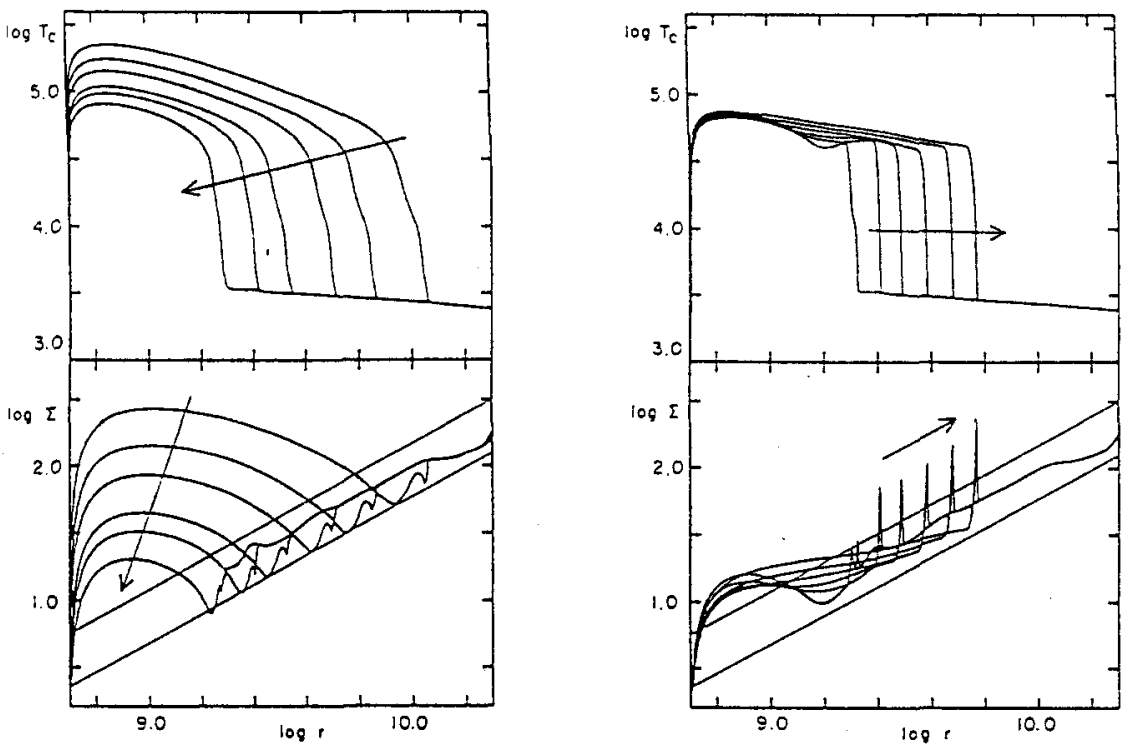

Fig. 5. Dynamics of transition wave propagation in dwarf novae. The left panels depict the time evolution in the central temperature (upper) and in surface density (lower) distribution in the disk in the decay phase, whereas the right panels display that in the rising phase.

pressure, $P_{\text {gas }} ; P_{\text {rad }}>P_{\text {gas }}$ (Lightman and Eardley 1974; Shibazaki and Hoshi 1975; Shakura and Sunyaev 1976). The fate of these instabilities are, however, still open to question at this moment; Shapiro et al. (1976) argued that the instabilities may lead to the formation of two-temperature, optically thin disks, while the slim disk model by Abramowicz et al. (1988) asserts that a third branch is realized by efficient advective cooling in the relativistic disks, so that the disk can undergo limit cycles, similarly to the case of DN or X-ray binaries (see $\S 3$ ).

Let us examine the latter model in some details. The thermal equilibrium curve is schematically depicted in Fig. 7. In $P_{\text {rad }}$ dominated disks, diffusion timescale $t_{\text {vis }}$ becomes comparable to the thermal timescales $t_{\text {th }}$, where

$$
t_{\mathrm{th}} \equiv \frac{C_{p} \Sigma T}{Q^{+}} \sim \frac{1}{\alpha \Omega}
$$

We thus have $t_{\text {vis }} \geq t_{\text {th }}$, so the disk trajectory in the $(\Sigma, \dot{M})$ plane is somewhat distinct from the case of DN (cf. Fig. 3). Evolutionary paths are also indicated in Fig. 7 by arrows. When point $\mathrm{C}$ is reached, $\dot{M}$ increases by the onset of the thermal instability. In the meantime, the value of $\Sigma$ decreases because of short viscous timescale. Detailed calculations have been performed 


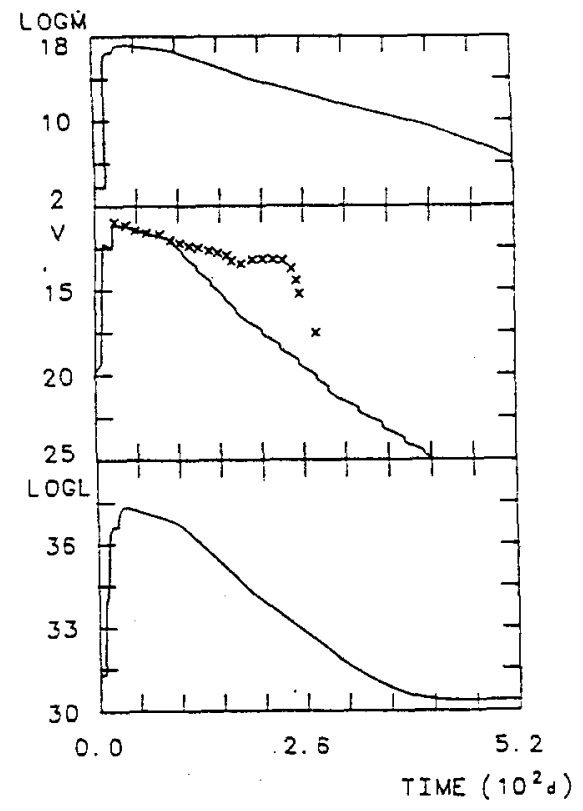

Fig. 6. Theoretical light curves of X-ray nova outbursts. From top, the mass-accretion rate at the inner edge of the disk, the $V$-magnitude, and the bolometric luminosity. Crosses represent the observed V-magnitudes of the black-hole nova A0620-00 by Whelan et al. (1977).

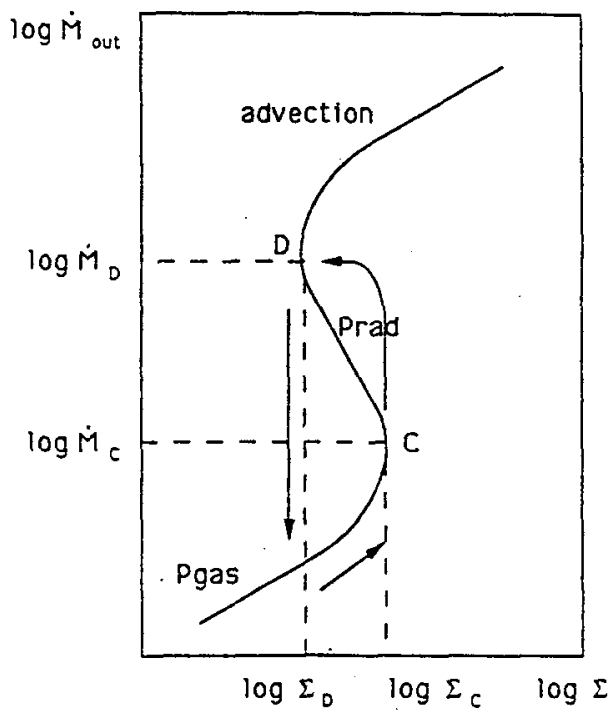

Fig. 7. Schematic thermal equilibrium curve in the slim disk model. The disk is gas pressure dominated on the lower branch, while it is radiation pressure dominated on the middle branch. The upper branch is also radiation pressure dominated, but advective cooling is substantial on this branch, which is distinct from the middle branch. The expected trajectory is schematically displayed by arrows. 


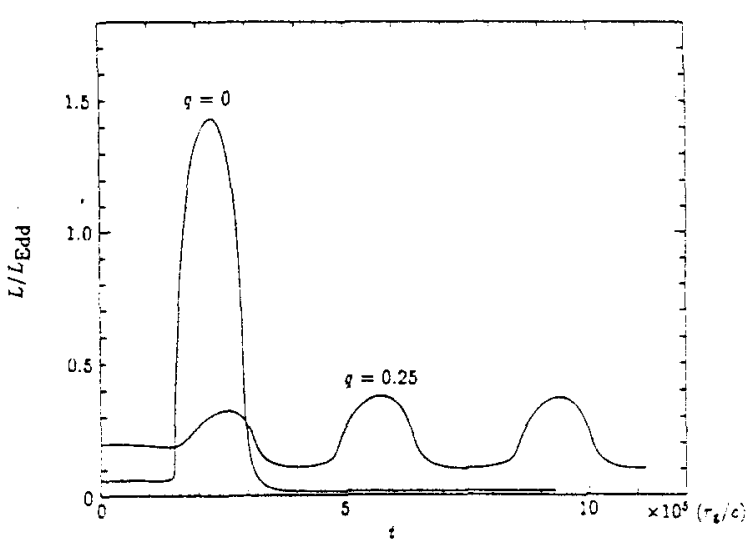

Fig. 8. Light curves of radiation pressure-dominated disks (due to courtesy of Honma et at. 1991). Here the $r_{\varphi}$ component of the shear stress tensor is prescribed as $\sigma_{r \varphi}=-\alpha P_{\mathrm{sot}} \beta^{q}$, where $\beta \equiv P_{\mathrm{gas}} / P_{\mathrm{tot}}$.

by Honma et al. (1991), who succeeded in reproducing limit cycle-type light curves as illustrated in Fig. 8.

There are some observational implications of these instabilities, which include quasi-periodic oscillation (van der Klis 1989), Type II bursts of Xray binaries (Lewin et al. 1976), and AGN activities. For details of these observational features, see a review by Matsuoka (1992) in this volume.

\subsection{Instabilities in Optically Thin Disks}

In optically thin disks, the following two process are substantial.

(a) Two-Temperature Plasmas

When a disk is optically thin, and is thus of low density, the disk plasma may be in a two-temperature state; electron temperature is less than proton temperature, $T_{\mathrm{p}}>T_{\mathrm{e}}$, by several orders of magnitudes. Reason for this can be explained as follows: In accretion process, a proton acquires more energy than an electron because of larger proton mass than the electron mass. Cooling of plasmas is, on the other hand, done mainly by electrons through bremsstrahlung and/or inverse-Compton cooling. In low density plasmas, coupling between protons and electrons is not strong if only Coulomb interaction is taken into account. Protons then cannot be cooled as quickly as electrons are.

By changing the efficiency of Coulomb coupling, the disk plasma may possibly alternate between a two-temperature state and a one-temperature state, thereby modulating hard X-ray intensities, although a specific mechanism for such transition is not yet known.

(b) $e^{+} e^{-}$Pair Generation

When the disk is optically thin, efficiency of plasma cooling is substan- 


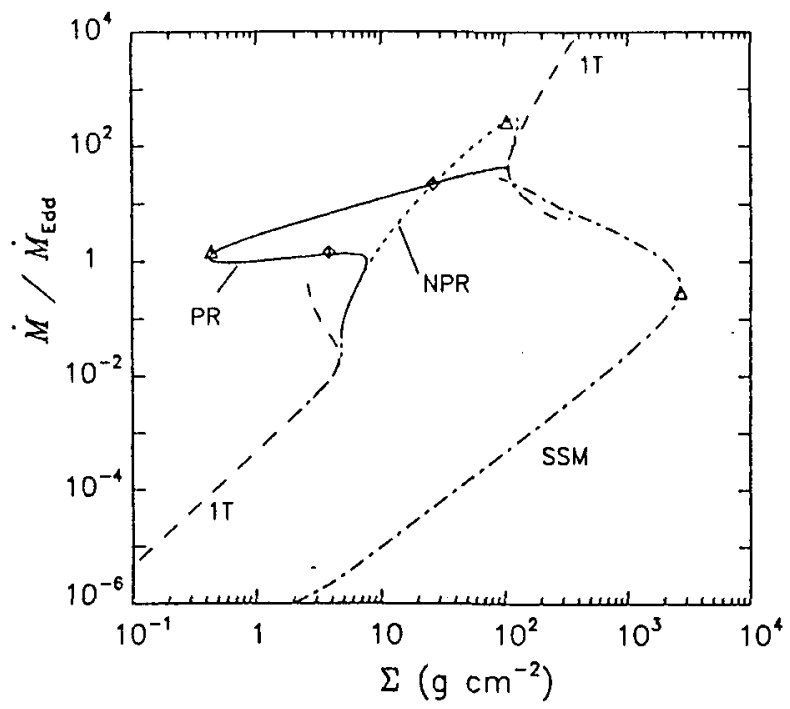

Fig. 9. Thermal equilibrium curves of one-temperature and two-temperature disks. One-temperature solutions are indicated by the dashed lines with a label " $1 \mathrm{~T}$ ", whereas the short-dash line labeled by NPR depicts the two-temperature solutions without $\mathrm{e}^{+} \mathrm{e}^{-}$production, and the solid line labeled by PR represents those with $\mathrm{e}^{+} \mathrm{e}^{-}$pairs. The dash-dot line corresponds to the modified Shakura-Sunyaev solution.

tially reduced. This, in turn, increases plasma temperatures (both $T_{e}$ and $T_{\mathrm{p}}$ ). When $T_{\mathrm{e}}$ increases up to the temperature corresponding to electron rest-mass energy, $k_{\mathrm{B}} T_{\mathrm{e}} \sim 511 \mathrm{keV}$ (where $k_{\mathrm{B}}$ is the Boltzmann constant), copious $e^{+} e^{-}$pairs will be generated.

Thermal equilibrium curves of hot accretion disks are illustrated in Fig. 9 for a one-temperature disk and a two-temperature disk. In this figure, the equilibrium solutions with and without $e^{+} e^{-}$pairs are indicated by the solid line and the dashed lines, respectively. We find that some portions of one-temperature, optically thin branches are thermally stable, while all the two-temperature, optically thin branches are thermally unstable. We also see that the equilibrium curves are substantially modified by the presence of $e^{+} e^{-}$pairs. Moreover, the disk equilibrium structure can be more clearly understood in the three dimensional $(\dot{M}, \Sigma, z)$ space, where $z=n_{+} / n_{p}$ is the ratio of the number density of positrons to that of protons.

In Fig. 10 we depict the cross-section of such three-dimensional equilibrium curves by fixing the value of $\Sigma$. The proton heating rate is equal to the proton cooling rate on the thermal equilibrium curves (dashed line), while pair generation rate balances with pair annihilation rate on the pair equilibrium curves (solid line). There are three pair-thermal equilibrium solutions for a range of $\Sigma$. They are all thermally unstable, whereas only the middle one is unstable against perturbations in $z$. We now find a possibility of 


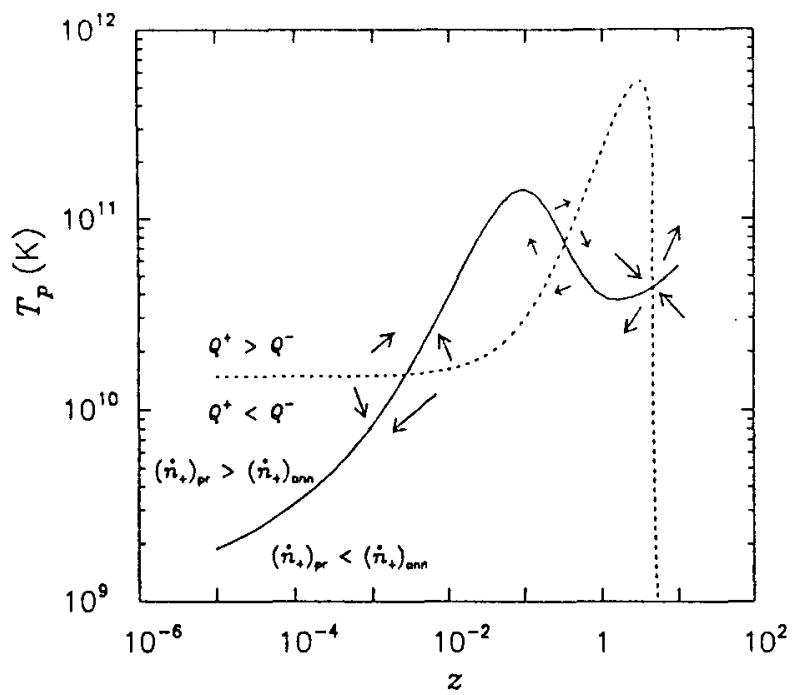

Fig. 10. Thermal equilibrium curve (by the short dashed line) and pair equilibrium curve (by the solid line) in the $\left(z, T_{\mathrm{p}}\right)$ plane. Three pair-thermal equilibrium solutions are found. Expected trajectories are also indicated by arrows. Limit cycles may be possible around the middle solution.

having limit-cycle behavior around the middle branch; When $T_{\mathrm{p}}$ increases, $z$ will also increase, because pair generation is more efficient at higher temperature. The number of coolants (i.e., electrons and positions) thus increases, which leads to rapid increment in the proton cooling rate, thereby decreasing $T_{\mathrm{p}}$. This, in turn, decreases the pair creation rate, resulting in decrease in the number of coolants and in proton cooling rate. The proton temperature again increases. Limit cycles as such may be responsible for rapid X-ray variabilities (flickering) in $\mathrm{XB}$ and $\mathrm{AGN}$.

In conclusion, optically thin disks are thermally unstable (Pringle et al. 1973), unless a disk plasma remains in a one-temperature state or unless $e^{+} e^{-}$pair production is taken into account (Kusunose and Mineshige 1992).

\subsection{IrRadiation-Induced Instabilities}

So far we considered as a heating source viscous heating only. In the case of X-ray binaries or active galactic nuclei (AGN), however, X-ray illumination of the outer portion of the disk by a central star or by the inner disk could dominate over viscous heating (SS73; Begelman et al. 1983). In such cases, overstable oscillation in gas flow may be induced by the irradiationinduced instability (Meyer and Meyer-Hofmeister 1984; Shields et al. 1986; Schwarzenberg-Czerny 1989).

When $\dot{M}_{\text {in }}$ increases, mass accretion rate through the inner edge of the disk, $\dot{M}_{\text {acc }}$, should also increase on the viscous timescales (see Fig. 11). 


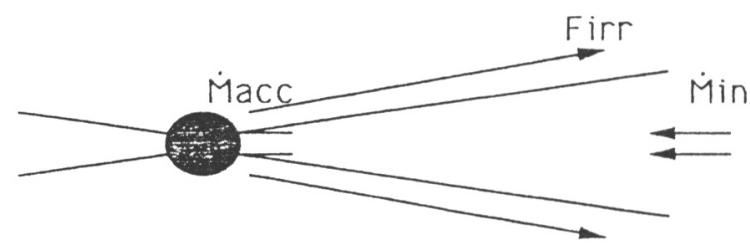

Fig. 11. A schematic view of an irradiated accretion disk. The mass accretion rate from the outer portions of the disk $\dot{M}_{\text {in }}$ is largely influenced by the presence of strong irradiating flux, $F_{\mathrm{irr}}$.

Enhancement in $\dot{M}_{\text {acc }}$ is likely to reinforce irradiating flux, $F_{\text {irr }}$, thereby causing further increase in $\dot{M}_{\text {in }}$. The irradiation thus works as positive feedback. As long as $\dot{M}_{\text {in }}$ and $\dot{M}_{\text {acc }}$ are kept large, gas in the outer portions of the disk will eventually be depleted. This will drastically reduce mass accretion rate and $F_{\text {irr }}$. We then have overstable oscillation under a certain condition (Shields et al. 1986). Chaotic behavior may also be produced (Bell et al. 1991).

\subsection{Radial Pulsational Instability}

The pulsational instability in stars is now well-known phenomenon, but little is known about radial pulsational instability in accretion disks. Kato (1978) was the first to investigate radial pulsations of accretion disks. Along this line, Bulmenthal et al. (1984) performed more detailed analysis.

In contrast to the stars, in which self-gravity force is balanced with pressure gradient force, centrifugal force, $r \Omega^{2}$, is balanced with the gravity due to a central star, $g=G M / r^{2}$, in accretion disks. When radial perturbation is added to a ring in equilibrium, the radius of the ring will oscillate around the equilibrium value because of epicyclic motion of orbiting gas elements.

The oscillation amplitudes will increase with time when $\alpha$ viscosity model is used. The amplifying mechanism is explained by two processes: the thermal process and the dynamical process. The thermal process is analogous to the $\epsilon$ mechanism for stellar pulsation (see a review by Saio 1992 in this volume). If energy generation rate, $\epsilon$, increases in a compressed phase, oscillation will acquire energy. Unlike the stellar case, in which energy generation is only available at the core where oscillation amplitudes are small, this mechanism really works well in the accretion disks, in which viscous heating is everywhere available.

Disk pulsation also acquires energy through the work done on a rotating ring by viscous stress. This is the dynamical process. As a result, disks are overstable against radial axisymmetric perturbations under a variety of conditions (Blumenthal et al. 1984). The results of nonlinear numerical simulations (cf. Okuda and Mineshige 1991) confirm the presence of global overstable modes (see Okuda and Mineshige 1992 in this volume). They are 
suspected to cause QPOs in CVs and low-mass X-ray binaries.

\subsection{Non-Radial Oscillations}

Disks are also unstable against non-axisymmetric perturbation. Kato (1983) discussed non-radial oscillations of accretion disks with $m=1$ mode (where $m$ is the azimuthal wave number) in Newtonian potential. Long-term $V / R$ variation of Be stars may be explained by this sort of one-armed oscillation (see Okazaki 1992 in this volume).

Kato (1989) further considered the non-radial oscillations in relativistic disks. He used the pseudo-Newtonian potential and found by the linear analysis some marginally overstable modes, in which horizontal motion is essential. One-armed oscillations of the disks in X-ray binaries is suggested to cause low frequency noise (LFN). For more details, please refer to the original papers.

\subsection{Tidal Instability}

Finally, we discuss the disk instabilities in more realistic potentials. The standard SS model assumes axisymmetric potential, and so neglect nonaxisymmetric effects, such as the tidal effect by a companion star, which could be important in outer portions of the disk in close binaries. When the mass ratio, $q=M_{2} / M_{1}<1 / 4$, where $M_{2}$ and $M_{1}$ are masses of a companion star and of a compact star, respectively, the orbital motion of a test particle around a central star becomes unstable at large radii (Paczyński 1974). Two-dimensional simulations demonstrate that the disk shape then becomes elliptical, and its semi-major axis gradually rotates in the inertial frame in the same direction as that of the orbital motion (Whitehurst 1988; Hirose and Osaki 1990). When the elongated rim of the disk is passed by the companion star, the disk luminosity is periodically enhanced due to excess energy dissipation by the tidal torque. The period of such light variation is slightly longer than the orbital period by a few percents. This is believed to explain so-called superhump light variations observed in superoutbursts of SU UMa stars, a subgroup of DN, and possibly in some XBs.

When superhump light variation is observed, it is possible to roughly estimate the mass of a compact star, $M_{1}$, from the empirical relation between $M_{1}$ and the superhump and orbital periods. Using this method, Mineshige et al. (1992) conclude that the compact components in X-ray novae, GS2000+25 and GS2023+338 (V404 Cyg), are very likely to be black holes instead of neutron stars.

A unified model for ordinary outbursts and superoutbursts in SU UMa system is discussed by Osaki (1989) (see also Osaki et al. (1992) in this volume.) 


\section{Summary}

We have briefly explained recent development of the theoretical studies on accretion disks, with emphasis on the disk instabilities. As we have seen previously, the current understanding of the accretion disks largely depend on the $\alpha$ viscosity hypothesis by Shakura and Sunyaev (1973). This SS model also assumes that disks are in stationary state; i.e., the same amount of gas is continuously put into the disk from the outer rim, and the exactly same amount of the gas is accreted onto a central star through the inner rim, so the disk luminosity $L_{\mathrm{d}}$ is kept constant in time. By relaxing some of the basic assumptions adopted in the standard SS model, we can derive various kinds of disk instabilities, which may be responsible for observable time variabilities on a wide rage of timescales.

In Table I, we summarize the basic assumptions of the SS disk and the relevant disk instabilities. It is very interesting to study global response of local instabilities, and to construct a more sophisticated time-dependent disk models, by which detailed comparison with observations is possible.

TABLE I

Disk Instabilities

\begin{tabular}{ccc}
\hline Standard Model & Time-Dependent Model & Observational Appearance \\
\hline HII (ionized) & HI $\leftrightarrow$ HII transition & DN, XN, AGN (?) \\
- & $P_{\text {Iad } \leftrightarrow P_{\text {gas }} \text { transition }}$ & QPO (?), AGN (?) \\
1-T (one-temperature) & $1-\mathrm{T} \leftrightarrow 2-T$ transition & high-low transition (?) \\
no pairs & $\gamma \gamma \leftrightarrow \mathrm{e}^{+} \mathrm{e}^{-}$transition & flickering (?) \\
no irradiation & irradiation-induced instability & AGN activity (?) \\
Kepler rotation & radial pulsation & QPO (?) \\
& nonradial pulsation & LFN (?) \\
axisymmetric & tidal instability & superhumps \\
\hline
\end{tabular}

Throughout this review, we neglect some other effects, which could be important; such as nonthermal emission processes and various kinds of magnetic activity. We also use the $\alpha$ model for disk viscosity. Unfortunately, the nature of the disk viscosity is poorly known, although fascinating models for creating large-magnitude viscosity in accretion disks are being proposed recently, which include shear instabilities (Drury 1977; Papaloizou and Pringle 1984), thermal convection (Lin and Papaloizou 1980), spiral shocks (Sawada et al. 1986), internal waves (Vishniac and Diamond 1989), magnetohydroturbulence (Ichimaru 1976; Kato and Horiuchi 1986), and magnetohydrodynamic (MHD) instabilities (Balbus and Hawley 1991). However at this moment, it is extremely difficult to develop alternative viscosity models because of complex behavior of hydrodynamic or magneto-hydrodynamic turbulence. 
Instead of seeking possible seeds for disk viscosity, one may use the theory of disk instabilities as a probe to refine the model for the disk viscosity through comparison with the observations. The thermal limit cycle instability (explained in $§ 3$ ) is, in this respect, the most successful case; in fact, many groups of people claim that $\alpha$ should be larger in HII state than in HI state. From the fitting light curves of DN, roughly we find

$$
\alpha \sim(0.05-0.2)\left[\left(\frac{T}{10^{4} \mathrm{~K}}\right)\left(\frac{r}{10^{10} \mathrm{~cm}}\right)\right]^{0.4-0.6},
$$

for a temperature range $3000 \leq T \leq 10^{5}(\mathrm{~K})$.

We thus finally wish to stress the importance of studying the timedependent properties of unstable accretion disks. This is also a useful way to get information on disk viscosity.

\section{Acknowledgements}

The author would like to thank Eiko Kawazoe for drawing figure 2, and Itoh Science Foundation for support.

\section{References}

Abramowicz, M. A., Czerny, B., Lasota, J. P., and Szuszkiewicz, E.: 1988, Astrophysical Journal 332, 646.

Alexander, D. R., Johnson, H. R., and Rypma, R. L.: 1983, Astrophysical Journal 272, 773.

Balbus, S. A., and Hawley, J. F.: 1991, Astrophysical Journal 376, 214.

Begelman, M. C., McKee, C. F., and Shields, G. A.: 1983, Astrophysical Journal 271, 70.

Bell, K. R., Lin, D. N. C., and Ruden, S. P.: 1991, Astrophysical Journal 372, 633.

Blumenthal, G. R., Yang, L. T., and Lin, D. N.: 1984, Astrophysical Journal 287, 771.

Cannizzo, J. K., Ghosh, P., and Wheeler, J. C.: 1982a, Astrophysical Journal 260, L83.

Cannizzo, J. K., Wheeler, J. C., and Ghosh, P.: 1982b, Pulsations in Classical and Cataclysmic Variables, eds. J. P. Cox and C. J. Hansen (Boulder, University of Colorado Press), p. 13.

Cannizzo, J. K., Wheeler, J. C., and Ghosh, P.: 1985, Proc. Cambridge Workshop on Cataclysmic Variables and Low-Mass X-ray Binaries, eds. D. Q. Lamb and J. Patterson (Dordrecht, Reidel), p. 307.

Cannizzo, J. K., Wheeler, J. C., and Polidan, R. S.: 1986, Astrophysical Journal 301, 634.

Cox, A. N., and Stewart, J. N.: 1970, Astrophysical Journal, Supplement Series 19, 243.

Cox, J. P., and Guili, R. T.: 1968, Principle of Stellar Structure (New York, Gordon and Breach).

Drury, L. O'C.: 1977, PhD. Thesis, Cambridge University.

Faulkner, J., Lin, D. N. C., and Papaloizou, J. C. B.: 1983, Monthly Notices of the RAS 205,359 .

Hirose, M. and Osaki, Y.: 1990, Publications of the ASJ, 42, 135.

Honma, F., Matsumoto, R., and Kato, S.: 1991, Publications of the ASJ 43, 147.

Hōshi, R.: 1979, Prog. Theor. Phys. 61, 1307.

Ichimaru, S: 1976, Astrophysical Journal 208, 701.

Kato, S.: 1978, Monthly Notices of the RAS 185, 629.

Kato, S.: 1983, Publications of the ASJ 35, 249. 
Kato, S.: 1989, Publications of the ASJ 41, 745.

Kato, S., and Horiuchi, T.: 1986, Publications of the ASJ 38, 313.

Kusunose, M., and Mineshige, S: 1992, Astrophysical Journal 390, in press.

Lewin, W. H. G. et al.: 1976, Astrophysical Journal 207, L95.

Lightman, A. P. and Eardley, D. M.: 1974, Astrophysical Journal 187, L1.

Lin, D. N. C., and Papaloizou, J. C. B.: 1980, Astrophysical Journal 191, 37.

Lin, D. N. C., and Papaloizou, J. C. B.: 1985, Protostars and Planets II (Tucson, University of Arizona Press).

Matsuoka, M.: 1992, this volume.

Meyer, F.: 1984, Astronomy and Astrophysics 131, 303.

Meyer, F., and Meyer-Hofmeister, E.: 1981, Astronomy and Astrophysics 104, L10.

Meyer, F., and Meyer-Hofmeister, E.: 1982, Astronomy and Astrophysics 106, 34.

Meyer, F., and Meyer-Hofmeister, E.: 1984, Astronomy and Astrophysics 140, L35.

Mineshige, S.: 1987, Astron. Space Sci. 130, 331.

Mineshige, S., Hirose, M., and Osaki Y.: 1992, Publications of the ASJ Letters 44, in press.

Mineshige, S., Kim, S.-W. and Wheeler, J. C.: 1990, Astrophysical Journal 358, L5.

Mineshige, S., and Osaki, Y.: 1983, Publications of the ASJ 35, 377.

Mineshige, S., and Osaki, Y.: 1985, Publications of the ASJ 37, 1.

Mineshige, S., and Shield, G. A: 1990, Astrophysical Journal 351, 47.

Mineshige, S., and Wheeler, J. C.: 1989, Astrophysical Journal 343, 241.

Nicolis, G., and Prigogine, I.: 1977, Self-Organization in Nonequilibrium Systems (John Wiley and Sons, New York).

Novikov, I. D. and Thorne, K. P.: 1974, Black Holes, Les Houches 1972, eds. C. DeWitt and B. S. DeWitt (Gordon and Breach, New York), p. 343.

Okazaki, A. T.: 1992, this volume.

Okuda, T. and Mineshige, S.: 1991, Monthly Notices of the RAS 249, 684 .

Okuda, T., and Mineshige, S.: 1992, this volume.

Osaki, Y.: 1974, Publications of the ASJ 26, 429.

Osaki, Y.: 1989, Publications of the ASJ 41, 1005.

Osaki, Y., Hirose, M., and Ichikawa, S.: 1992, this volume.

Paczynski, B.: 1969, Acta Astron. 19, 1.

Paczynski, B.: 1977, Astrophysical Journal 216, 822.

Papaloizou, J., Faulkner, J., and Lin, D. N. C.: 1983, Monthly Notices of the RAS 205, 487.

Papaloizou, J. C. B., and Pringle, J. E.: 1984, Monthly Notices of the RAS 208, 721.

Pringle, J. E.: 1981, Annual Review of Astronomy and Astrophysics 19, 137.

Pringle, J. E., and Rees, M. J.: 1972, Astronomy and Astrophysics 21, 1.

Pringle, J. E., Rees, M. J., and Pacholczyk, A. G.: 1973, Astronomy and Astrophysics 29, 179.

Saio, H.: 1992, this volume.

Sawada, K., Matsuda, T., Hachisu, I.: 1986, Monthly Notices of the RAS 219, 75.

Schwarzenberg-Czerny, A.: 1989, Astronomy and Astrophysics 210, 174.

Shakura, N. I., and Sunyaev, R. A.: 1973, Astronomy and Astrophysics 24, 337.

Shakura, N. I., and Sunyaev, R. A.: 1976, Monthly Notices of the RAS 175, 613.

Shapiro, S. L., Lightman, A. P., and Eardley, D. M.: 1976, Astrophysical Journal 204, 187.

Shibazaki, N., and Hosi, R.: 1975, Prog. Theor. Phys. 54, 706.

Shields, G. A., McKee, C. F., Lin, D. N. C., and Begelman, M. C.: 1986, Astrophysical Journal 306, 90.

Smak, J.: 1982a, Acta Astron. 32, 199.

Smak, J.: 1982b, Acta Astron. 32, 213.

Smak, J.: 1984a, Publications of the ASP 96, 5.

Smak, J.: 1984b, Acta Astron. 34, 161.

Tainaka, K., Fukazawa, S., and Mineshige, S.: 1992, this volume.

Van der Klis, M.: 1989, Annual Review of Astronomy and Astrophysics 27, 517.

Vishniac, E. T., and Diamond, P.: 1989, Astrophysical Journal 347, 447. 
Whelan, J. A. J., et al.: 1977, Monthly Notices of the RAS 180, 657.

Whitehurst, R.: 1988, Monthly Notices of the RAS 232, 55. 